\title{
Pigmentläsion: Lasertherapie mit bitteren Folgen
}

\author{
Essener Dermatologen erinnern daran, dass Melanome der Haut gerade dort entstehen können, \\ wo zuvor Pigmentläsionen per Laser entfernt worden sind, und das bis zu zehn Jahre danach.
}

Es gibt bisher nur wenige Berichte, meist Kasuistiken, im Zusammenhang mit Melanomen, die nach dem Entfernen von Pigmentläsionen mithilfe eines Lasers entstanden sind. Dermatologen der Universität Regensburg haben 2006 darauf hingewiesen, dass es seit 1999 immer wieder solche Berichte gab (Acta Derm Venereol 2006;86:44-7). Nun berichten Dr. Sarah Delker von der Klinik für Dermatologie der Uniklinik Essen über elf Patienten - sechs Frauen und fünf Männer -, die zwischen 2007 und 2011 am dortigen Hautkrebszentrum wegen eines Melanoms behandelt wurden, das nach der Laserbehandlung einer Pigmentläsion entstanden war. Die Patienten waren zwischen 42 und 72 Jahre alt (im Mittel 58 Jahre). Zwischen Lasertherapie und Melanomdiagnose lagen durchschnittlich drei Jahre und vier Monate (mindestens 0,8 und maximal zehn Jahre).

Nur bei zwei Patienten wurde vor der Laserbehandlung eine Biopsie für eine histologische Untersuchung entnommen. Bei dem einen Patienten diagnostizierten die Ärzte eine nävoide Lentigo, bei dem anderen Patienten einen dysplastischen Nävus. Bei sechs Patienten erfolgte die Laserbehandlung nur einmal, bei vier Patienten mehrfach, bevor die Läsion exzidiert wurde. Alle Laserbehandlungen sind von zertifizierten Dermatologen

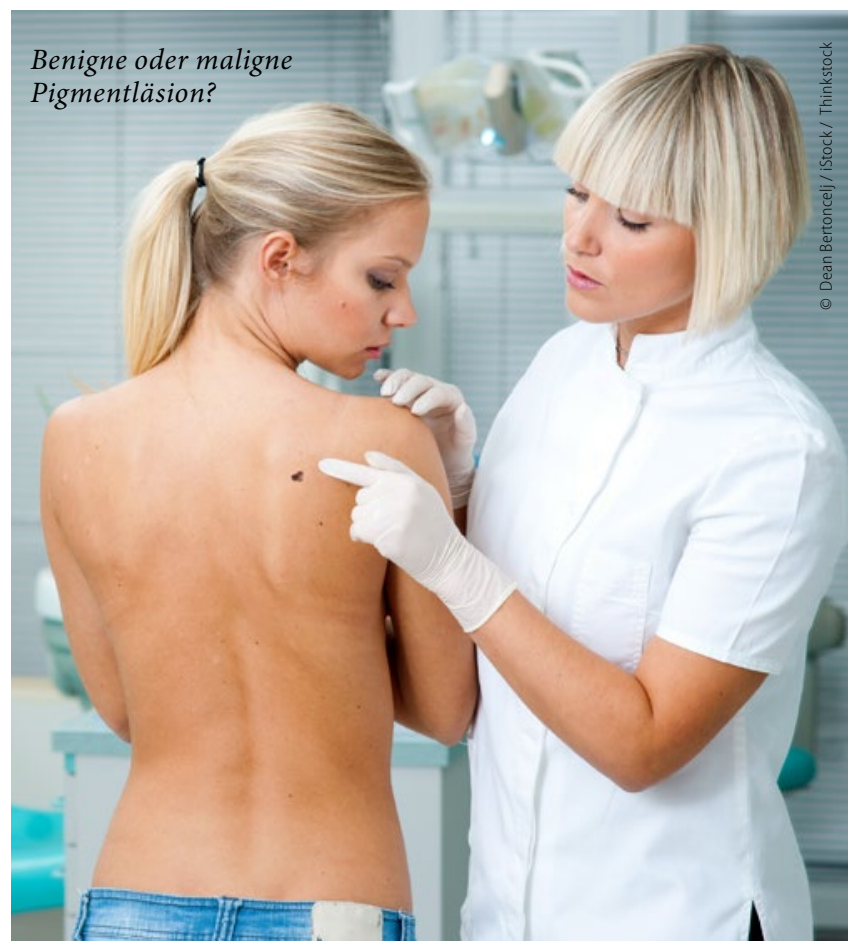

vorgenommen worden. Das spreche dagegen, dass solche Fehldiagnosen nur von dermatologisch ungeübten Ärzten gemacht würden, so Delker und ihre Kollegen. Sie erinnern daran, dass auch erfahrene Dermatologen in nur $80 \%$ der Fälle allein anhand morphologischer Kriterien benigne von malignen Pigmentläsionen unterscheiden können.

\section{3-Jähriger erkrankt am Melanom und stirbt}

Von den elf Patienten starb einer an den Folgen einer Melanomerkrankung. Bei dem 53-jährigen Mann war auf dem Rücken drei Jahre vor der Diagnose eine Laserbehandlung wegen einer Pigmentläsion vorgenommen worden. Die Essener Ärzte diagnostizierten dann 2010 histologisch Makrometastasen in den rechten axillären Lymphknoten, die mithilfe einer radikalen Lymphadenektomie entfernt wurden. Es stellte sich heraus, dass zwei von acht Lymphknoten positiv waren. Kurze Zeit nach der Diagnose wurden per CT Lungenmetastasen entdeckt. In der Folge entwickelten sich trotz Therapie auch Hirnmetastasen, fünf Monate später kam es zur Krankheitsprogression. Auch eine Behandlung mit Temozolomid konnte dem Patienten nicht mehr helfen. Die übrigen Patienten ließen sich hingegen rechtzeitig erfolgreich behandeln.

Die Kasuistik macht deutlich, dass eine durch Fehlbeurteilung verspätete Diagnose einen Melanoms, wenn es bereits zur Metastasierung gekommen ist, tödlich enden kann. Zudem können nach der Laserbehandlung von Pigmentläsionen Rezidive entstehen. Weil zuvor keine Biopsien gemacht wurden, sondern man sich auf eine makroskopische Beurteilung beschränkt hatte, blieben möglicherweise Melanome unentdeckt. Wie groß das Problem tatsächlich ist, lässt sich nach Ansicht der Essener Ärzte anhand ihrer Kasuistiken schwer abschätzen. Weil ihre Klinik eine hochspezialisierte Einrichtung für Melanompatienten hat, ist möglichweise die Zahl der Patienten mit Melanomen nach Laserbehandlung höher als in anderen Regionen. Dennoch halten es die Ärzte für möglich, dass solche Kasuistiken mit zunehmender Anwendung der Laserbehandlung bei Pigmentläsionen häufiger werden. Patienten sollten darüber informiert werden, dass eine Laserbehandlung möglicherweise tieferliegende Melanozyten nicht erreichen kann und in der Folge benigne oder aber auch maligne Rezidive entstehen können. Delker und ihre Kollegen empfehlen, bei Pigmentläsionen auf eine Laserbehandlung ganz zu verzichten und stattdessen komplett zu exzidieren und das Gewebe histopathologisch zu untersuchen. Peter Leiner therapy. J Dermatol 2016, online 27. Juni; doi: 10.1111/1346-8138.13484 